\title{
Chronological Survey
}

1.1 Pisces (since kambrium, silur, ca. 800-600 million years)

1.2 Amphibia (since perm, 350 million years)

1.3 Reptiles (since trias, jura, 250 million years)

1.4 Aves (cretaceus period, since 180 million years)

1.5 Mammalia (since tertiary, 70 million years)

1.5.1 Monotremata (ovipara, ornitorhyncus)

1.5.2 Marsupialia (vivipara, e.g., kangaroo)

1.5.3 Placentalia since 60 million years

1.5.3.1 Insectivora (shrew mouse, Erynaceus, mole, chiroptera)

1.5.3.2 Apatidae since ca. 80 million years

1.5.3.2.1 Prosimiae (e.g., Tupaja glis) since ca. 60 million years

1.5.3.2.2 Simiae

1.5.3.2.3 Hominoides since ca. 22 million years

1.5.3.2.3.1 Orang utan since ca. 16 million years

1.5.3.2.3.2 Gorilla since ca. 7 million years

1.5.3.2.3.3 Chimpanzee since ca. 6 million years

1.5.3.2.3.4 Homo since 2.5-2 million years

1.5.3.2.3.4.1 Homo rudolfensis ("Lucy")

1.5.3.2.3.4.2 Homo habilis

1.5.3.2.3.4.3 Homo ergaster

1.5.3.2.3.4.4 Homo erectus and others

1.5.3.2.3.4.4.1 Homo heidelbergensis

1.5.3.2.3.4.4.2 Homo sapiens (one common species)

1.5.3.2.3.4.4.2.1 Homo steinheimensis ca. 350,000 years

1.5.3.2.3.4.4.2.2 Homo neanderthalensis ca. 200,000-30,000 years

1.5.3.2.3.4.4.2.3 Homo recens (Diluvium-Alluvium) since ca. 30,000 years

1.5.3.3 Rodentia

1.5.3.4 Carnivora

1.5.3.5 Ungulata

1.5.3.6 Cetacea (whales) 